\title{
Quantum teleportation of photonic qudits using linear optics
}

\author{
Chenyu Zhang $\odot,{ }^{1,2}$ J. F. Chen, ${ }^{3}$ Chaohan Cui, ${ }^{4,2,5,6}$ Jonathan P. Dowling, ${ }^{7,8,9,10}$ Z. Y. Ou, ${ }^{11,12}$ and Tim Byrnes ${ }^{2,1,9,13,14, *}$ \\ ${ }^{1}$ State Key Laboratory of Precision Spectroscopy, School of Physical and Material Sciences, \\ East China Normal University, Shanghai 200062, China \\ ${ }^{2}$ New York University Shanghai, 1555 Century Ave, Shanghai 200122, China \\ ${ }^{3}$ Shenzhen Institute for Quantum Science and Engineering and Department of Physics, \\ Southern University of Science and Technology, Shenzhen 518055, China \\ ${ }^{4}$ College of Optical Sciences, University of Arizona, Tucson, Arizona 85718, USA \\ ${ }^{5}$ CAS Key Laboratory of Quantum Information, University of Science and Technology of China, Hefei 230026, China \\ ${ }^{6}$ Synergetic Innovation Center of Quantum Information \& Quantum Physics, University of Science and Technology of China, \\ Hefei, Anhui 230026, China \\ ${ }^{7}$ Hearne Institute for Theoretical Physics, Department of Physics and Astronomy, Louisiana State University, \\ Baton Rouge, Louisiana 70803, USA \\ ${ }^{8}$ CAS-Alibaba Quantum Computing Laboratory, USTC, Shanghai 201315, China \\ ${ }^{9}$ NYU-ECNU Institute of Physics at NYU Shanghai, 3663 Zhongshan Road North, Shanghai 200062, China \\ ${ }^{10}$ National Institute of Information and Communications Technology, 4-2-1, Nukui-Kitamachi, Koganei, Tokyo 184-8795, Japan \\ ${ }^{11}$ Department of Physics, Indiana University-Purdue University Indianapolis, 402 North Blackford Street, Indianapolis, Indiana 46202, USA \\ ${ }^{12}$ Quantum Institute of Light and Atoms, Department of Physics, East China Normal University, Shanghai 200241, China \\ ${ }^{13}$ National Institute of Informatics, 2-1-2 Hitotsubashi, Chiyoda-ku, Tokyo 101-8430, Japan \\ ${ }^{14}$ Department of Physics, New York University, New York, New York 10003, USA
}

(Received 3 July 2019; published 23 September 2019)

\begin{abstract}
One of the challenges of photon-based quantum teleportation is that both a source of entangled photons and an entangled basis measurement are required. For qubits, one can perform a probabilistic entangled basis measurement using linear optics, making the scheme efficient. However, for photonic qudits, an equivalent scheme remains difficult to devise. In this paper, we generalize the probabilistic photonic qubit teleportation protocol to qudits. The method relies on producing permutation entangled states nondeterministically which are superpositions of permutations of the spatial and qudit states of the photons. Our scheme nondeterministically teleports a photonic qudit using only entangled photon sources, linear optics, and photon detectors, and should be experimentally realizable for small qudit dimensions.
\end{abstract}

DOI: 10.1103/PhysRevA.100.032330

\section{INTRODUCTION}

Quantum teleportation is of central importance in the field of quantum information technology not only from a foundational perspective [1,2], but also because of its numerous applications such as quantum computing [3-5] and quantum cryptography [6-10]. Experimentally it was first realized with polarization encoded photonic qubits $[11,12]$. Photonic quantum teleportation has been improved and performed over increasingly longer distances since its first realization [13-24], with the current record at $1400 \mathrm{~km}$ [25]. In particular, Marcikic, Gisin, and co-workers realized time-bin encoded teleportation, which has the advantage that it is robust over long distances [26-28]. The largest multiqubit teleportation that has been demonstrated to date is for two qubits, encoded by the polarization of two photons [29] and the orbital and polarization degrees of freedom of one photon [30]. Qubit teleportation has been proposed and realized in other physical

*tim.byrnes@nyu.edu systems, such as with trapped atoms [31-34] and solid-state systems [35,36].

For higher-dimensional systems (i.e., qudits), extensions of the original teleportation protocol have been performed [37-39]. Continuous variable teleportation [40] was realized first in optical systems [41-45] and was also performed with atomic ensembles [46]. Numerous proposals for qudit teleportation have been proposed in the past [47-58]. Many variations of high-dimensional teleportation, such as those using multiphoton encodings [59], spin coherent states [60-62], and telecloning $[63,64]$, have been proposed. However, qudit teleportation has been experimentally challenging. One reason for this is that qudit teleportation schemes often rely on the presence of a nonlinearity at some point in the protocol; either implicitly or explicitly. In the case of photons, since no strong nonlinear medium is available, this makes the implementation problematic.

In this paper, we propose a qudit teleportation scheme for photons using only linear optics, entangled-photon sources, and photon detectors. Specifically we aim to teleport qudits consisting of a single photon, implemented using time binning, for example. This rules out the use of multiqubit 
teleportation [37,39] or multiphoton encodings [59]. The central part of our protocol is a nondeterministic method of measuring in an entangled basis. This generalizes the photonic scheme described and implemented by Refs. [11,65,66], where a Bell measurement is realized without nonlinear elements. Our fully linear optical-measurement scheme allows for the qudit version of the Bell measurement, and entangled state preparation necessary for teleportation. Our linear-optical implementation makes our scheme much more experimentally viable than currently proposed schemes. We note that recently Goyal, Konrad, and co-workers provided an approach to the problem by using determinant statesentangled states of light which use completely antisymmetric photon states [54,67]. Although the scheme uses only linear optics in the teleportation protocol itself, the preparation of the determinant states requires nonlinear optical devices, which suppress two-photon populations, thereby adding demands to the experimental implementation. Another difficulty of the implementation of their work is that the teleportation scheme is achievable only with the antisymmetric entangled state, which gives a extremely low success rate in the state preparation step. Our scheme overcomes several of these difficulties.

\section{TELEPORTATION SCHEME}

The complete teleportation scheme, including state preparation, is shown in Fig. 1. The aim of the procedure is to teleport a photonic qudit on the mode $a_{0 i}$ in Alice's possession, to mode $a_{N i}$ in Bob's possession. Here the mode $a_{n i}$ has two labels, the first for the spatial mode $n \in[0, N]$ and the second for the qudit state $i \in[1, N]$. The qudit can be implemented for example using time bins, such that the $i$ labels each time bin. The input state to be teleported is written

$$
|\psi\rangle=\sum_{i_{0}=1}^{N} \psi_{i_{0}} a_{0 i_{0}}^{\dagger}|0\rangle .
$$

The modes $a_{1 i_{1}}$ to $a_{N i_{N}}$ are prepared in an entangled state consisting of permutations of the spatial and qudit states (the permutation-entangled state), which can be prepared in a nondeterministic way. The modes $a_{0 i_{0}}$ through mode $a_{N i_{N}}$ are then interfered by a linear-optical network performing a unitary operation $U$ on the modes. After the output of the optical network is measured, the results are fed forward, and a local operation is performed. At the end of this process, the initial state (1) is teleported to mode $a_{N i_{N}}$.

We can prove that this procedure works using the following general argument. First we must define the permutationentangled state as a photonic state where every qudit label from $i \in[1, N]$ is present and every spatial mode $n \in[1, N]$ is involved. The detection will destroy the photons in the state preparation box of Fig. 1, but their entangled partner will survive in this state and go to Alice's box. The general form of this state can be written

$$
|E\rangle=\sum_{i \in S_{N}} \phi_{i_{1} \ldots i_{N}} \prod_{n=1}^{N} a_{n i_{n}}^{\dagger}|0\rangle,
$$

where the sum runs over all elements $\boldsymbol{i}=\left(i_{1}, \ldots, i_{N}\right)$ of the symmetric group $S_{N}$, i.e., all permutations of the numbers $1,2, \ldots, N$, and $\phi$ is a normalized wave function. Of course,

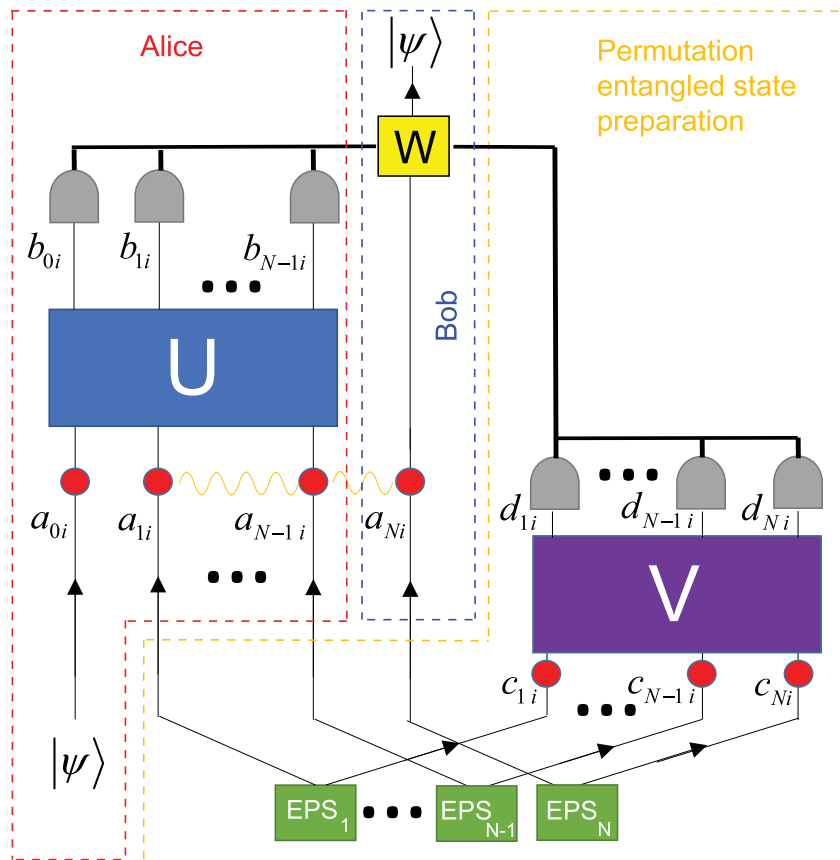

FIG. 1. Teleportation scheme. Thin lines are the paths of the photons labeled by modes $a_{n i}, b_{n i}, c_{n i}, d_{n i}$ where $n$ are spatial mode labels and $i$ are qudit labels. The qudit may be realized by time bins labeled by $i$, for example. Thick lines correspond to classical commutation channels. The parts of the apparatus in possession by Alice (the sender), Bob (the receiver), and the permutation entangled state preparation are indicated. Wiggly lines denote entanglement of the permutation entangled state. Photons start at $N$ entangled photon sources (EPS) and enter linear optical networks $V$ and $U$ and are detected. The detector results are fed forward and a local unitary operation $W$ is performed. At the end, the state on mode $a_{0 i}$ is teleported to mode $a_{N i}$.

not all choices of $\phi_{i_{1} \ldots i_{N}}$ result in a $12 \ldots N$ entangled state, but writing it in this form will allow us to handle the general case from which we can derive a criterion. The initial state at the starting point of the teleportation is thus $|\psi\rangle|E\rangle$. We now measure in a permutation-entangled basis, which takes the general form

$$
|M\rangle=\sum_{j \in S_{N}} \chi_{j_{0} \ldots j_{N-1}} \prod_{n=0}^{N-1} a_{n j_{n}}^{\dagger}|0\rangle,
$$

where $\boldsymbol{j}=\left(j_{0}, \ldots, j_{N-1}\right)$ and $\chi$ is a wave function defining the measurement basis. Details on how this measurement can be achieved will be discussed below. A measurement in this basis then results in the unnormalized state

$$
\begin{aligned}
P_{M}|\psi\rangle \otimes|E\rangle= & \sum_{i_{0} \ldots i_{N}=1}^{N}\left|\epsilon_{i_{1} \ldots i_{N}}\right|\left|\epsilon_{i_{0} \ldots i_{N-1}}\right| \\
& \times \psi_{i_{0}} \phi_{i_{1} \ldots i_{N}} \chi_{i_{0} i_{1} \ldots i_{N-1}} a_{N i_{N}}^{\dagger}|0\rangle,
\end{aligned}
$$

where $P_{M}=|M\rangle\langle M|$ is the projection operator for the state (3). We have written $|E\rangle$ and $|M\rangle$ in the equivalent form $|E\rangle=$ $\sum_{i_{1} \ldots i_{N}=1}^{N}\left|\epsilon_{i_{1} \ldots i_{N}}\right| \phi_{i_{1} \ldots i_{N}} a_{1 i_{1}}^{\dagger} \ldots a_{N i_{N}}^{\dagger}|0\rangle$, where $\epsilon_{i_{1} \ldots i_{N}}$ is the LeviCivita antisymmetric tensor, and similarly for $|M\rangle$. Using the identity that $\left|\epsilon_{i_{1} \ldots i_{N}}\right|\left|\epsilon_{i_{0} \ldots i_{N-1}}\right|=\delta_{i_{0} i_{N}}\left|\epsilon_{i_{1} \ldots i_{N}}\right|$, we can write the 
unnormalized measured state as

$$
P_{M}|\psi\rangle \otimes|E\rangle=\sum_{i_{N}=1}^{N} f\left(i_{N}\right) \psi_{i_{N}} a_{N i_{N}}^{\dagger}|0\rangle,
$$

where

$$
f\left(i_{N}\right)=\sum_{i_{1} \ldots i_{N-1}=1}^{N}\left|\epsilon_{i_{1} \ldots i_{N}}\right| \phi_{i_{1} \ldots i_{N}} \chi_{i_{N} i_{1} \ldots i_{N-1}} .
$$

Equation (5) is clearly closely related to the original state, up to the function $f$. If it is possible to perform a unitary operation $W$ such that $W a_{N i}^{\dagger} W^{\dagger}=a_{N i}^{\dagger} / f(i)$ on the state (5), such that $f$ is removed, then the teleportation is successful. Since the only diagonal unitary matrices are phase operations, the criterion for a successful teleportation is

$$
|f(i)|=f_{0} \quad \forall i,
$$

where $f_{0}>0$ is a nonzero constant. The probability of obtaining this result is given by the amplitude of the state (5), which is $p_{\text {tel }}=f_{0}^{2}$.

\section{MEASUREMENT IN THE PERMUTATION-ENTANGLED BASIS}

We now show how to measure in the basis $|M\rangle$ using only linear optics and photon detectors. The measurement is discussed first because the same scheme is used in the preparation step. Our measurement scheme generalizes the well-known trick of measuring in the Bell basis using a beamsplitter $[11,65,66]$. Consider the linear optical network $U$ in Fig. 1. By preparing (preselecting) initial states that have one photon in the input spatial modes $a_{n i}$ and postselecting all results where there is a result in every qudit state (e.g., time bin), an effective measurement in the basis (3) can be constructed. To show this, start from the output mode and write the final detected state as

$$
\left|B_{r}\right\rangle=\prod_{j=1}^{N} b_{r(j) j}^{\dagger}|0\rangle .
$$

Here $r(j)$ is the spatial mode label of the $j$ th detection result. This fully specifies the detection outcome for the class of postselected states. The linear optical network transforms the input and output modes according to [68]

$$
b_{n j}=\sum_{m=0}^{N-1} u_{n m} a_{m j} .
$$

The detected state is then

$$
\left|B_{r}\right\rangle=\sum_{m_{1} \ldots m_{N}=0}^{N-1} \prod_{j=1}^{N} u_{r(j) m_{j}}^{*} a_{m_{j} j}^{\dagger}|0\rangle .
$$

This state involves terms where there is more than one photon in a spatial mode. However, such states do not exist, according to the initial state preparation, where exactly one photon is input to the network per spatial mode. We thus separate the terms involving one photon per mode as

$$
\begin{aligned}
\left|B_{r}\right\rangle & =\left|B_{r}^{(1)}\right\rangle+\left|B_{r}^{(\perp)}\right\rangle, \\
\left|B_{r}^{(1)}\right\rangle & =\sum_{m_{1} \ldots m_{N}=0}^{N-1}\left|\epsilon_{m_{1} \ldots m_{N}}\right| \prod_{j=1}^{N}\left(u_{r(j) m_{j}}^{*} a_{m_{j} j}^{\dagger}\right)|0\rangle, \\
\left|B_{r}^{(\perp)}\right\rangle & =\sum_{m_{1} \ldots m_{N}=0}^{N-1}\left(1-\left|\epsilon_{m_{1} \ldots m_{N}}\right|\right) \prod_{j=1}^{N}\left(u_{r(j) m_{j}}^{*} a_{m_{j} j}^{\dagger}\right)|0\rangle .
\end{aligned}
$$

As long as the input state to the linear network involves only one photon per spatial mode, the overlap with $\left|B_{r}^{(\perp)}\right\rangle$ will be zero. The state $\left|B_{r}^{(1)}\right\rangle$ contains all permutations of the spatial labels, weighted with the factors originating from the linear transformation matrix. This can be relabeled in terms of the spatial mode labels instead of the qudit (e.g., time bin) labels

$$
\left|B_{r}^{(1)}\right\rangle=\sum_{j \in S_{N}} \prod_{n=0}^{N-1} u_{r\left(j_{n}\right) n}^{*} a_{n j_{n}}^{\dagger}|0\rangle .
$$

This has the same form as (3) with $\chi_{j_{0} \ldots j_{N-1}}=\prod_{n=0}^{N-1} u_{r\left(j_{n}\right) n}^{*}$, and hence a measurement in the permutation-entangled basis is achieved as long as the input state has one photon per mode, and the output is postselected on the state with one photon per qudit state (e.g., time bin). Another way to view (12) is that it is the state that is measured through a bosonsampling network, with a coefficient corresponding to the matrix permanent of the linear network $U$ [69]. Note that the photons do not need to be distributed in the output state as one photon per mode. For example, $r(j)=1$ corresponding to all photons exiting the first spatial mode is allowable.

\section{PREPARATION OF THE PERMUTATION-ENTANGLED STATE}

We adapt the measurement technique above to prepare the desired entangled state (2). The basic strategy is to start in a multiphoton-entangled state, and perform a measurement in the permutation-entangled state as described in (12). This then leaves the unmeasured photons in a permutation-entangled state ready for use in the teleportation. The initial state after the emission of the entangled photon sources is

$$
\left|\Omega_{0}\right\rangle=\prod_{n=1}^{N}\left(\frac{1}{\sqrt{N}} \sum_{i_{n}=1}^{N} a_{n i_{n}}^{\dagger} c_{n i_{n}}^{\dagger}\right)|0\rangle .
$$

The $c_{n j}$ photons then enter the linear optical network $V$ which gives a transformation

$$
d_{n i}=\sum_{m=1}^{N} v_{n m} c_{m i},
$$

and is measured and postselected on events where each photon occupies a different qudit state $i$. Using the same argument as that arriving at (11), the detected state can be written

$$
\begin{gathered}
\left|D_{s}\right\rangle=\prod_{i=1}^{N} d_{s(i) i}^{\dagger}|0\rangle=\left|D_{s}^{(1)}\right\rangle+\left|D_{s}^{(\perp)}\right\rangle, \\
\left|D_{s}^{(1)}\right\rangle=\sum_{i \in S_{N}} \prod_{n=1}^{N} v_{s\left(i_{n}\right) n}^{*} c_{n i_{n}}^{\dagger}|0\rangle,
\end{gathered}
$$


and $\left|D_{s}^{(\perp)}\right\rangle$ is again a state which contains more than one photon per mode in the input state and hence is guaranteed to have zero overlap with (13). Projecting $P_{D_{s}}=\left|D_{s}\right\rangle\left\langle D_{s}\right|$ onto (13) we have

$$
P_{D_{s}}\left|\Omega_{0}\right\rangle=\left|D_{s}\right\rangle \otimes \frac{1}{\sqrt{N^{N}}} \sum_{i \in S_{N}} \prod_{n=1}^{N} v_{s\left(i_{n}\right) n} a_{n i_{n}}^{\dagger}|0\rangle .
$$

This has the same form as (2) with $\phi_{i_{1} \ldots i_{N}}=$ $\left(\prod_{n=1}^{N} v_{s\left(i_{n}\right) n}\right) / \sqrt{p_{\text {prep }}(s)}$, which shows that the teleportation can be performed using the scheme in Fig. 1.

\section{SUCCESS PROBABILITY}

For a successful teleportation, one requires preparation of a permutation-entangled state of the form (17). This requires an output state such that there is one $d_{n j}$ photon per qudit state (e.g., time bin). The probability of a given outcome $s(i)$ is given by the amplitude of (17)

$$
p_{\text {prep }}(s)=\frac{1}{N^{N}} \sum_{i \in S_{N}} \prod_{n=1}^{N}\left|v_{s\left(i_{n}\right) n}\right|^{2} .
$$

Given that a suitable permutation-entangled state has been prepared, a successful teleportation again requires one $b_{n i}$ photon per qudit state (e.g., time bin) and the condition (7) satisfied. The probability for the outcomes that (7) are satisfied are

$$
p_{\text {tel }}(r \mid s)=\frac{\left|v_{s(N) N} u_{r(N) 0}^{*}\right|^{2}}{N^{N} p_{\text {prep }}(s)}\left|\sum_{i \in S_{N-1}} \prod_{n=1}^{N-1} v_{s\left(i_{n}\right) n} u_{r\left(i_{n}\right) n}^{*}\right|^{2},
$$

here $\boldsymbol{i}=\left(i_{1}, \ldots, i_{N-1}\right)$ and we have set $i_{N}=N$, since $|f(i)|^{2}$ is assumed to be a constant. Then the probability of a successful teleportation given a $r(i)$ and $s(i)$ is $p_{\text {suc }}(r, s)=$ $p_{\text {tel }}(r \mid s) p_{\text {prep }}(s)$.

The above expressions clearly depend upon the particular linear optical networks $U, V$ that are used. We now evaluate the performance of the teleportation for particular examples of linear optical networks. The best performing network we have found is the quantum Fourier transform

$$
u_{n m}=v_{n+1 m+1}=\frac{1}{\sqrt{N}} e^{\frac{2 \pi i n m}{N}} .
$$

This reduces to standard photonic qubit teleportation for $N=2$. For a given optical network the total preparation or teleportation probability is then found by running through all possible outcomes $r(i)$ and $s(i)$ and adding the probabilities for which a successful result is obtained.

We show the success probability for various $N$ in Table I. For $N=2$ we recover the same success probability for the usual teleportation, as achieved in Ref. [11]. For $N=2$, preparation of the permutation-entangled state is not necessary, as demonstrated in Ref. [11]. The permutation-entangled state involves $N$ photons, and hence for $N>2$, the state preparation as given in Fig. 1 is necessary. As expected, the success probability goes down as $N$ increases. This is a result of the lack of nonlinear media to prepare and detect
TABLE I. Probabilities for permutation-entangled state preparation and teleportation. (a) Probability of preparation $\sum_{s} p_{\text {prep }}(s)$ using (18) where the sum runs over all possible $s(i)$. (b) Probability of a successful teleportation $\sum_{r} p_{\text {tel }}(r \mid s)$ using (19) where only those cases satisfying (7) are counted. The preparation state uses the fixed outcome $s(i)=i$. For all calculations, the quantum Fourier transform (20) is used for $U$ and $V$ and bracketed values denote equivalent values to two decimal places.

\begin{tabular}{cccccc}
\hline \hline$N$ & 2 & 3 & 4 & 5 & 6 \\
\hline (a) & $1 / 2(0.5)$ & $2 / 9(0.22)$ & $3 / 32(0.09)$ & $24 / 125(0.19)$ & $5 / 324(0.02)$ \\
(b) & $1 / 2(0.5)$ & $1 / 9(0.11)$ & $5 / 32(0.15)$ & $1 / 25(0.04)$ & $1 / 18(0.06)$ \\
\hline \hline
\end{tabular}

the permutation-entangled state. For very large $N$ the scheme clearly does not scale well. However, at the several-photon level, the success probability for both preparation and teleportation is at the $\sim 1 \%$ level, which is well within experimental realization. The overall success probability is then found by adding $p_{\text {suc }}(r, s)$ for all possible $r(i)$ and $s(i)$ that satisfy $(7)$. Calculating the results for $2 \leqslant N \leqslant 4$ we find that the results are identical to the product of the preparation and teleportation probabilities in Table I.

\section{EXPERIMENTAL IMPLEMENTATION}

We now discuss some of the experimental details to implement Fig. 1. For the entangled photon sources (EPSs), traditional parametric down-conversion (PDC) crystals produce paired photon states with a very short coherence time, usually in the range of $\approx \mathrm{fs}$. This will complicate the state preparation of the permutation-entangled state, since the photons must enter the linear optical network $V$ within the same coherence time window. An alternative to PDC is to use entangled photons from atomic clouds, which have coherence times of more than 10 ns [70-72]. To create the multiphoton-entangled state required by (13), a pair of continuous wave laser beams should be applied onto an atomic cloud to induce spontaneous four-wave mixing, in which the entangled paired photons are generated [73-75]. Therefore the initial entangled photon state requires synchronization of photon pairs into the same time window of $10 \mathrm{~ns}$ or more, emitted by multiple atomic clouds. The generation rate of two pairs of entangled photons, emitted from independent atomic clouds within a $\Delta T=10 \mathrm{~ns}$ window, is typically $p_{\text {emit }}^{2}=0.01 / \mathrm{s}$ [76], where $p_{\text {emit }}$ is the generation rate for one photon occupying a specific time bin with length of $\Delta T$. In order to align $N$ entangled photons, there is a total generation rate of $p_{\text {emit }}^{N}=0.1^{N} / \mathrm{s}$. Thus the total success probability including the emission probability will be the product of $p_{\text {emit }}^{N}$, with the probabilities in Table I. In practice, the preparation of the entangled photons will be incorporated into the postselection process, where only outcomes involving $N$ of the $d_{n i}$ photons are counted.

\section{CONCLUSIONS}

We have proposed a photonic qudit-teleportation scheme using only linear optics, entangled photon sources, and photon detectors. The teleportation scheme is based on permutation- 
entangled states which mix the spatial and qudit states creating a multiphoton entangled state. The permutationentangled states are a class of states that are broader than matrix-permanent states, which are best known in the context of boson sampling. The entangled basis measurement is performed by postselecting particular outcomes after a linearoptical transformation. This generalizes the probabilistic Bell measurement that was the key to performing photonic qubit teleportation [11]. While the success probability is found to degrade rather quickly with the number of photons, we view this as a natural consequence of not using a nonlinearity in the measurement - the nondeterministic nature is the price to be paid for not using a genuine nonlinearity. At the level of several photons the probabilities are well within the range of current experimental capabilities.

Note added in proof: Recently we became aware of Ref. [77] where experimental qutrit teleportation was achieved and a scheme for high-dimensional photonic teleportation was proposed.

\section{ACKNOWLEDGMENTS}

The authors thank Alexei Gilchrist for discussions. T.B. is supported by the Shanghai Research Challenge Fund; New York University Global Seed Grants for Collaborative Research; National Natural Science Foundation of China (61571301,D1210036A); the NSFC Research Fund for International Young Scientists (11650110425,11850410426); NYU-ECNU Institute of Physics at NYU Shanghai; the Science and Technology Commission of Shanghai Municipality (17ZR1443600); the China Science and Technology Exchange Center (NGA-16-001); and the NSFC-RFBR Collaborative grant (81811530112). J.F.C is supported by the National Natural Science Foundation of China (11674100), the Natural Science Foundation of Shanghai No. 16ZR1448200, and Shanghai Rising-Star Program 17QA1401300. J.P.D. would like to acknowledge support from the U.S. Air Force Office of Scientific Research, Army Research Office, the Defense Advanced Research Projects Agency, and the National Science Foundation.
[1] C. H. Bennett, G. Brassard, C. Crépeau, R. Jozsa, A. Peres, and W. K. Wootters, Phys. Rev. Lett. 70, 1895 (1993).

[2] S. Pirandola, J. Eisert, C. Weedbrook, A. Furusawa, and S. L. Braunstein, Nat. Photon. 9, 641 (2015).

[3] G. Brassard, S. L. Braunstein, and R. Cleve, Physica D: Nonlinear Phenom. 120, 43 (1998).

[4] D. Gottesman and I. L. Chuang, Nature 402, 390 (1999).

[5] A. I. Lvovsky, B. C. Sanders, and W. Tittel, Nat. Photon. 3, 706 (2009).

[6] H.-J. Briegel, W. Dür, J. I. Cirac, and P. Zoller, Phys. Rev. Lett. 81, 5932 (1998).

[7] E. Waks, A. Zeevi, and Y. Yamamoto, Phys. Rev. A 65, 052310 (2002).

[8] B. C. Jacobs, T. B. Pittman, and J. D. Franson, Phys. Rev. A 66, 052307 (2002).

[9] W. K. Wootters and W. H. Zurek, Nature 299, 802 (1982).

[10] R. F. Werner, Phys. Rev. A 58, 1827 (1998).

[11] D. Bouwmeester, J.-W. Pan, K. Mattle, M. Eibl, H. Weinfurter, and A. Zeilinger, Nature 390, 575 (1997).

[12] D. Boschi, S. Branca, F. De Martini, L. Hardy, and S. Popescu, Phys. Rev. Lett. 80, 1121 (1998).

[13] Y.-H. Kim, S. P. Kulik, and Y. Shih, Phys. Rev. Lett. 86, 1370 (2001).

[14] R. Ursin, T. Jennewein, M. Aspelmeyer, R. Kaltenbaek, M. Lindenthal, P. Walther, and A. Zeilinger, Nature 430, 849 (2004).

[15] X.-M. Jin, J.-G. Ren, B. Yang, Z.-H. Yi, F. Zhou, X.-F. Xu, S.-K. Wang, D. Yang, Y.-F. Hu, S. Jiang et al., Nat. Photon. 4, 376 (2010).

[16] J. Yin, J.-G. Ren, H. Lu, Y. Cao, H.-L. Yong, Y.-P. Wu, C. Liu, S.-K. Liao, F. Zhou, Y. Jiang et al., Nature 488, 185 (2012).

[17] X.-S. Ma, T. Herbst, T. Scheidl, D. Wang, S. Kropatschek, W. Naylor, B. Wittmann, A. Mech, J. Kofler, E. Anisimova et al., Nature 489, 269 (2012).

[18] B. J. Metcalf, J. B. Spring, P. C. Humphreys, N. Thomas-Peter, M. Barbieri, W. S. Kolthammer, X.-M. Jin, N. K. Langford, D. Kundys, J. C. Gates et al., Nat. Photon. 8, 770 (2014).
[19] J.-W. Pan, M. Daniell, S. Gasparoni, G. Weihs, and A. Zeilinger, Phys. Rev. Lett. 86, 4435 (2001).

[20] T. Jennewein, G. Weihs, J.-W. Pan, and A. Zeilinger, Phys. Rev. Lett. 88, 017903 (2001).

[21] E. Lombardi, F. Sciarrino, S. Popescu, and F. De Martini, Phys. Rev. Lett. 88, 070402 (2002).

[22] D. Fattal, E. Diamanti, K. Inoue, and Y. Yamamoto, Phys. Rev. Lett. 92, 037904 (2004).

[23] R. Ursin, F. Tiefenbacher, T. Schmitt-Manderbach, H. Weier, T. Scheidl, M. Lindenthal, B. Blauensteiner, T. Jennewein, J. Perdigues, P. Trojek et al., Nat. Phys. 3, 481 (2007).

[24] P. Kok and S. L. Braunstein, Phys. Rev. A 61, 042304 (2000).

[25] J.-G. Ren, P. Xu, H.-L. Yong, L. Zhang, S.-K. Liao, J. Yin, W.-Y. Liu, W.-Q. Cai, M. Yang, L. Li et al., Nature 549, 70 (2017).

[26] R. T. Thew, S. Tanzilli, W. Tittel, H. Zbinden, and N. Gisin, Phys. Rev. A 66, 062304 (2002).

[27] I. Marcikic, H. De Riedmatten, W. Tittel, H. Zbinden, and N. Gisin, Nature 421, 509 (2003).

[28] H. de Riedmatten, I. Marcikic, W. Tittel, H. Zbinden, D. Collins, and N. Gisin, Phys. Rev. Lett. 92, 047904 (2004).

[29] Q. Zhang, A. Goebel, C. Wagenknecht, Y.-A. Chen, B. Zhao, T. Yang, A. Mair, J. Schmiedmayer, and J.-W. Pan, Nat. Phys. 2, 678 (2006).

[30] X.-L. Wang, X.-D. Cai, Z.-E. Su, M.-C. Chen, D. Wu, L. Li, N.-L. Liu, C.-Y. Lu, and J.-W. Pan, Nature 518, 516 (2015).

[31] M. Riebe, H. Häffner, C. Roos, W. Hänsel, J. Benhelm, G. Lancaster, T. Körber, C. Becher, F. Schmidt-Kaler, D. James et al., Nature 429, 734 (2004).

[32] M. Barrett, J. Chiaverini, T. Schaetz, J. Britton, W. Itano, J. Jost, E. Knill, C. Langer, D. Leibfried, R. Ozeri et al., Nature 429, 737 (2004).

[33] S. Olmschenk, D. Matsukevich, P. Maunz, D. Hayes, L.-M. Duan, and C. Monroe, Science 323, 486 (2009).

[34] S. Ritter, C. Nölleke, C. Hahn, A. Reiserer, A. Neuzner, M. Uphoff, M. Mücke, E. Figueroa, J. Bochmann, and G. Rempe, Nature 484, 195 (2012). 
[35] W. Gao, P. Fallahi, E. Togan, A. Delteil, Y. Chin, J. MiguelSanchez, and A. Imamoğlu, Nat. Commun. 4, 2744 (2013).

[36] F. Bussières, C. Clausen, A. Tiranov, B. Korzh, V. B. Verma, S. W. Nam, F. Marsili, A. Ferrier, P. Goldner, H. Herrmann et al., Nat. Photon. 8, 775 (2014).

[37] M. S. Zubairy, Phys. Rev. A 58, 4368 (1998).

[38] S. Stenholm and P. J. Bardroff, Phys. Rev. A 58, 4373 (1998).

[39] G. Rigolin, Phys. Rev. A 71, 032303 (2005).

[40] S. L. Braunstein and H. J. Kimble, Quantum Information with Continuous Variables (Springer, Berlin, 1998), pp. 67-75.

[41] A. Furusawa, J. L. Sørensen, S. L. Braunstein, C. A. Fuchs, H. J. Kimble, and E. S. Polzik, Science 282, 706 (1998).

[42] H. Yonezawa, T. Aoki, and A. Furusawa, Nature 431, 430 (2004).

[43] N. Lee, H. Benichi, Y. Takeno, S. Takeda, J. Webb, E. Huntington, and A. Furusawa, Science 332, 330 (2011).

[44] W. P. Bowen, N. Treps, B. C. Buchler, R. Schnabel, T. C. Ralph, H.-A. Bachor, T. Symul, and P. K. Lam, Phys. Rev. A 67, 032302 (2003).

[45] S. Babichev, J. Ries, and A. Lvovsky, Europhys. Lett. 64, 1 (2003).

[46] H. Krauter, D. Salart, C. Muschik, J. M. Petersen, H. Shen, T. Fernholz, and E. S. Polzik, Nat. Phys. 9, 400 (2013).

[47] Z. Xiao-Gui, L. Hong-Mei, and Z. Hao-Sheng, Chin. Phys. Lett. 23, 2900 (2006).

[48] A. K. Pati and P. Agrawal, Phys. Lett. A 371, 185 (2007).

[49] L. Xi-Han, D. Fu-Guo, and Z. Hong-Yu, Chin. Phys. Lett. 24, 1151 (2007).

[50] Z. Zhang, Y. Liu, and D. Wang, Phys. Lett. A 372, 28 (2007).

[51] J. Dong and J. Teng, Eur. Phys. J. D 49, 129 (2008).

[52] M. Al-Amri, J. Evers, and M. S. Zubairy, Phys. Rev. A 82, 022329 (2010).

[53] K. Marshall and D. F. V. James, J. Opt. Soc. Am. B 31, 423 (2014).

[54] S. K. Goyal, P. E. Boukama-Dzoussi, S. Ghosh, F. S. Roux, and T. Konrad, Sci. Rep. 4, 4543 (2014).

[55] R. F. Werner, J. Phys. A 34, 7081 (2001).

[56] T. Mor and P. Horodecki, arXiv:quant-ph/9906039.

[57] W. Son, J. Lee, M. S. Kim, and Y.-J. Park, Phys. Rev. A 64, 064304 (2001).
[58] Y.-J. Gu, Y.-Z. Zheng, and G.-C. Guo, Phys. Lett. A 296, 157 (2002).

[59] A. Halevy, E. Megidish, T. Shacham, L. Dovrat, and H. S. Eisenberg, Phys. Rev. Lett. 106, 130502 (2011).

[60] A. N. Pyrkov and T. Byrnes, New J. Phys. 16, 073038 (2014).

[61] T. Byrnes, D. Rosseau, M. Khosla, A. Pyrkov, A. Thomasen, T. Mukai, S. Koyama, A. Abdelrahman, and E. Ilo-Okeke, Opt. Commun. 337, 102 (2015).

[62] A. N. Pyrkov and T. Byrnes, Phys. Rev. A 90, 062336 (2014).

[63] F. Grosshans and P. Grangier, Phys. Rev. A 64, 010301(R) (2001).

[64] P. van Loock and S. L. Braunstein, Phys. Rev. Lett. 87, 247901 (2001).

[65] S. L. Braunstein and A. Mann, Phys. Rev. A 51, R1727 (1995).

[66] N. Lütkenhaus, J. Calsamiglia, and K.-A. Suominen, Phys. Rev. A 59, 3295 (1999).

[67] A. Miranowicz, J. Opt. B 7, 142 (2005).

[68] M. Reck, A. Zeilinger, H. J. Bernstein, and P. Bertani, Phys. Rev. Lett. 73, 58 (1994).

[69] S. Aaronson and A. Arkhipov, in Proceedings of the Forty-Third Annual ACM Symposium on Theory of Computing (ACM, New York, 2011) pp. 333-342.

[70] V. Balić, D. A. Braje, P. Kolchin, G. Y. Yin, and S. E. Harris, Phys. Rev. Lett. 94, 183601 (2005).

[71] B. Srivathsan, G. K. Gulati, B. Chng, G. Maslennikov, D. Matsukevich, and C. Kurtsiefer, Phys. Rev. Lett. 111, 123602 (2013).

[72] J. F. Chen and S. Du, Front. Phys. 7, 494 (2012).

[73] S. Du, P. Kolchin, C. Belthangady, G. Y. Yin, and S. E. Harris, Phys. Rev. Lett. 100, 183603 (2008).

[74] Z. Han, P. Qian, L. Zhou, J. F. Chen, and W. Zhang, Sci. Rep. 5, 9126 (2015).

[75] C. Shu, P. Chen, T. K. A. Chow, L. Zhu, Y. Xiao, M. M. Loy, and S. Du, Nat. Commun. 7, 12783 (2016).

[76] P. Qian, Z. Gu, R. Cao, R. Wen, Z. Y. Ou, J. F. Chen, and W. Zhang, Phys. Rev. Lett. 117, 013602 (2016).

[77] Y.-H. Luo, H.-S. Zhong, M. Erhard, X.-L. Wang, L.-C. Peng, M. Krenn, X. Jiang, L. Li, N.-L. Liu, C.-Y. Lu, A. Zeilinger, and J.-W. Pan, Phys. Rev. Lett. 123, 070505 (2019). 